\title{
La construcción de confianza para el estudio de prácticas de resistencia en la escolarización de jóvenes en contextos de pobreza urbana
}

\author{
Eduardo Langer ${ }^{1}$ \\ UNSAM/UNPA/CONICET
}

Recibido: 16-08-2016

Aceptado: 13-10-2016

\section{RESUMEN}

El objetivo es realizar una reflexión sobre la construcción metodológica realizada en una investigación doctoral que tuvo como finalidad describir prácticas de resistencia en la escolarización de jóvenes, en escuelas secundarias en contextos de pobreza urbana. La preocupación por las técnicas de investigación con jóvenes en instituciones y en contextos particulares, remite a una pregunta por los modos en los que el investigador se relaciona con los sujetos, así como con las maneras de habitar y estar en las escuelas. Es como parte de esa inquietud que este artículo se propone caracterizar las maneras en las que se accede al conocimiento de esas prácticas diarias de los sujetos, a través de un diseño metodológico con estrategias múltiples de obtención de información y una articulación de métodos de investigación social. Para ello, en primer lugar se plantea una discusión en torno a los desafíos metodológicos en el marco de la sociología de la educación, que aporten ideas sobre la relación entre discursos y prácticas, sobre la significación en el nivel de la reflexibilidad de los sujetos. En segundo lugar, se trabaja sobre una de las estrategias que se desarrolló en la investigación mediante la construcción de confianza a largo plazo y comprometida con estudiantes y docentes en una escuela secundaria. A modo de cierre se propone que esta estrategia devenida en técnica de investigación, es una forma desde la cual pensar e intervenir en torno a la desigualdad educativa y social.

Palabras clave: Confianza, prácticas, jóvenes, escolarización, contextos de pobreza urbana. 


\title{
The construction of trust for the study of resistance practices in the schooling of young people in poor urban contexts
}

\begin{abstract}
The aim to this article is analyse the methodological process that has been implemented in a Doctoral research oriented to describe resistance practices to young students in high schools that are situated in poverty -urban contexts. Reflections on research methods to work with young students that belong to specifics contexts, are related to the ways the researcher interacts with the students and the ways to stay in the school. Starting from this, the article sets the ways to create knowledge to this daily practices through a methodological process with multiple strategies to collect information. In first place the article sets a discussion around methodological challenges in the Sociology of Education as discipline, highlighting the relationships between practices and discourses. In second place, it develops one of the methodological strategy implemented in the research that we can call building trust with students and teachers in scholar contexts. The article concludes that this methodological strategy converted to a research method is a resource to think and act around educative and social inequity.
\end{abstract}

Keywords: confidence - practices - young boys - schooling - context of urban poverty.

\section{Introducción}

En este trabajo se realizará una reflexión sobre algunas de las estrategias principales puestas en juego en una investigación doctoral, que tuvo como finalidad describir prácticas de resistencia en la escolarización de jóvenes, en escuelas secundarias en contextos de pobreza urbana, a través de las acciones diarias de oposición, lucha y contestación de los individuos en sus haceres y decires, tanto dentro como fuera del aula. 
La preocupación por las técnicas de investigación con jóvenes en instituciones y en contextos particulares, remite a una pregunta por los modos en los que el investigador se relaciona con los sujetos, así como con las maneras de habitar y estar en las escuelas, con las acciones y las interacciones que allí suceden. Es como parte de esa inquietud que este artículo se propone caracterizar las maneras en las que se accede al conocimiento de esas prácticas diarias de los sujetos a través de un diseño metodológico con estrategias múltiples de obtención de la información y una articulación de métodos de investigación social.

El diseño de investigación desarrollado combinó técnicas cualitativas y cuantitativas en un proceso de interacción entre diferentes componentes (Maxwell, 1996), y de una forma flexible a lo largo de todo el proceso, evaluando las implicancias de los propósitos, la teoría, las preguntas de investigación, los métodos y la empiria. Para ello, fue central explicitar en el diseño de la investigación los propósitos y las preguntas, las teorías antecedentes y el marco conceptual desde el cual se trabajó la noción de resistencia en contextos de pobreza urbana. En la investigación doctoral desarrollada se desplegó una "articulación de los métodos no convencionales de investigación social (instancias participativas de investigación y técnicas colectivas de obtención y análisis de la información) con métodos convencionales de investigación, cuantitativos y cualitativos" (Sirvent, 1999, p. 140), a través de un diseño metodológico de investigación que implicó la elaboración de una encuesta a estudiantes, trabajo de observación de clases, entrevistas flash y en profundidad, entrevistas a grupos de estudiantes y discusión de resultados de investigación con directores y docentes. Fue una investigación que abarcó un punto de vista arqueológico en tanto se ejerció "en una multiplicidad de registros" (Foucault, 2007, p. 205). Con este diseño se posibilitó la convergencia de modelos metodológicos, entendiendo a la metodología como el conjunto de procedimientos para confrontar teoría y empiria (Sirvent, 1999). En este trabajo, se propone reflexionar sobre algunos de los principios que este diseño de investigación implicó y no sobre las técnicas mismas.

Para ello, en primer lugar se plantean algunas discusiones sobre los desafíos metodológicos en el marco de la sociología de la educación, en procura de innovaciones para acceder a la construcción de conocimientos que aporten ideas sobre la relación entre discursos y prácticas, sobre la significación en el nivel de la reflexibilidad de los sujetos. En segundo lugar, se trabaja sobre la estrategia que se desarrolló en la investigación 
mediante la construcción de confianza a largo plazo y comprometida con estudiantes y docentes en una escuela secundaria.

\section{Desafíos metodológicos en el marco de la sociología de la educación}

El desafío metodológico desde el ámbito de la sociología de la educación y ante una estructura socioeconómica desigual e injusta en interacción con la heterogeneidad de procesos de construcción de subjetividades, es realizar análisis críticos en relación con algunas perspectivas teóricas ${ }^{2}$ que dan cuenta de la imposibilidad, la carencia o el déficit en los procesos de escolarización en el siglo XXI. Como dice Arroyo (2001), hay procesos de constitución de sujetos y hay generación de proyectos propios; de forma tal, que se vuelve central recolocar los vínculos entre educación, pobreza y sujetos en nuevas matrices para recuperar las pugnas y luchas por los derechos por parte de los sujetos y de las instituciones.

Ahora bien, ¿qué significa pensar en nuevas matrices desde una perspectiva de la sociología de la educación crítica? "¿Cuál es el significado y la naturaleza del concepto de crítico en la teoría crítica?" (Torres, 2008, p. 141). La sociología de la educación crítica requiere trabajar con diversas dimensiones y surge para entender la educación, el curriculum, la enseñanza, las prácticas áulicas y las políticas educativas. Sin embargo, la sociología de la educación crítica no procura sólo comprender sino, también, apunta a la transformación social o, como dice Torres (2008), "una perspectiva crítica de la sociología de la educación apunta a estudiar el poder y las relaciones de autoridad en la educación, y los fundamentos y consecuencias políticas para las políticas educativas" (Torres, 2008, p. 142). De hecho, una teoría crítica del poder y del Estado es un punto de partida necesario para estudiar el proceso de toma de decisiones educativas (Torres, 2008).

Estos aportes son importantes, ya que si bien las características sociolaborales, económicas y educativas contribuyen a producir subjetividades en la actualidad para reproducir y mantener las condiciones de la vida social, esos procesos de construcción de subjetividades y de generación de proyectos de vida se siguen sosteniendo en el espacio tradicional que tenía la escuela como eje integrador a otros ámbitos, tales como

2. Tal como las pedagogías de las competencias, las teorías del bullying o las del fin de la escuela y/o de desinstitucionalización. 
la cultura, el arte, los medios de comunicación u otras organizaciones sociales.

De esta manera, aquí importa discutir algunas de las implicancias centrales para el pensamiento socioeducativo de abordar un objeto de estudio desde el marco de la sociología de la educación crítica y a través de un registro y diseño múltiple de investigación ya que, de esta forma, se puede arrojar nueva luz sobre una serie de antinomias que son constitutivas del pensamiento político, social y educativo. Es decir, a partir de esos elementos, el diseño que se presenta en la investigación doctoral enfocada desde la sociología de la educación, se trata de discutir las formas de superar viejas y anticuadas antinomias, para comprender los actuales procesos complejos en el ámbito escolar. Para Miller y Rose (1990), las grandes dicotomías -micro y macro, lo público y privado, Estado y sociedad civil, estructura y acción, lo social y lo individual y, finalmente, la dominación y libertad- no son de gran utilidad. Hay relaciones indirectas entre regulación y persuasión no identificables con estas dicotomías, ellas continúan pensando al poder vs. la resistencia, lo individual vs. lo colectivo, lo público vs. lo privado, lo político vs. lo personal, como pares opuestos y omiten la medida en la cual, el yo no es personal, sino que es producto de relaciones de poder y el resultado de estrategias y tecnologías desarrolladas para crear la participación de la ciudadanía (Cruikshank, 1996).

Con el diseño de investigación que se desarrolló en la investigación, se intenta aportar a la reconfiguración en los debates sobre estas antinomias dominantes por mucho tiempo, para pensar el ejercicio del poder político y entender las formas contemporáneas de gobierno (Rose y Miller, 1992). La crítica a estas antinomias, además de ofrecer un marco epistemológico de debate, se vuelve central a la hora de diseñar la investigación, porque nos permite pensar en el tipo y grado de regularidad que estas acciones pueden tener en función de las coacciones relacionales en las que se producen las prácticas, que ésta investigación tuvo como objeto.

Las prácticas de resistencia no son producto exclusivo del lugar escuela y tienenjustificativos, antecedentesyconsecuenciasqueprovienendellugar, del tiempo y del espacio. Es decir, del mundo cultural. Para comprender y dar sentido a estas prácticas se involucran técnicas cuantitativas y cualitativas que permitieron trabajar sobre las particularidades en el contexto general en el que se desarrollan, siempre teniendo en cuenta que los significados y los sentidos que los sujetos dan a sus acciones, 
sólo pueden comprenderse junto a aquellos que los producen (Mallimaci y Salvia, 2005). Poder caracterizar las prácticas de resistencia desde las vivencias y las formas culturales de los jóvenes en contextos de pobreza urbana, posibilitó comprender cómo las sociedades son "cada vez más heterogéneas, fragmentadas y desiguales" (Kuasñosky y Szulik, 1995, p. 169). A la vez, percibir sus formas de vida, cómo entienden el mundo, cuáles son sus formas y expresiones políticas, qué creen que va a pasar en el futuro y sus expectativas sobre él, sus ideas sobre la escuela y la relación con el mundo del trabajo. Estas dimensiones fueron algunas de las más importantes que surgieron al escuchar a los jóvenes.

En suma, se estudiaron problemas, procesos, interacciones y situaciones que la vida de los sujetos iban expresando a través de sus perspectivas, sus historias, sus comportamientos, sus experiencias, sus acciones y sus sentidos, de forma situada en el contexto particular en el que tenían lugar (Vasilachis de Gialdino, 2007). Por ello, las regularidades que se expresaban en la vida cotidiana y desde los significados que los actores atribuían a esas interacciones (Vasilachis de Gialdino, 2007) se sucedieron de formas, por momentos, contradictorias. Aún con estas contradicciones, aquí se insiste en la caracterización de los aspectos positivos de esas culturas (Grignon y Passeron, 1991), sus aspectos creativos y las potencias de las acciones de los sujetos, ya que nos permiten enfatizar los aspectos productivos de estas relaciones e interacciones. Así, las prácticas de resistencia, en tanto discurso, remiten a un contexto y permiten desmontar falacias del pensamiento dominante o de los medios de comunicación, tales como: que a los jóvenes nada les importa o interesa, no quieren hacer nada, no esperan nada del futuro.

Teniendo en cuenta este marco, a continuación se desarrolla la reflexión sobre una de las estrategias metodológicas principales que se puso en marcha a partir de las diversas técnicas de investigación.

\section{La confianza para el estudio de las prácticas de los jóvenes en las escuelas}

El objeto de la investigación desarrollada fue caracterizar las prácticas de resistencia de estudiantes en contextos de pobreza urbana. Entonces, el principal criterio de selección fue el lugar donde se desarrolló el trabajo en profundidad, su emplazamiento en una región de asentamientos y 
villas miseria ${ }^{3}$, que desde fines de los años setenta ha tenido un constante crecimiento poblacional y territorial, que presenta importantes marcas de la pobreza urbana. Es decir, a sólo 200 metros de una villa miseria y a 600 de otra. Así lo expresa el mapa realizado por estudiantes de la escuela, en función de las propias ideas que tenían del territorio, de su barrio y de su escuela:

Mapa $\mathbf{N}^{\circ}$ 1: ubicación de la escuela en estudio con respecto a dos villas miseria

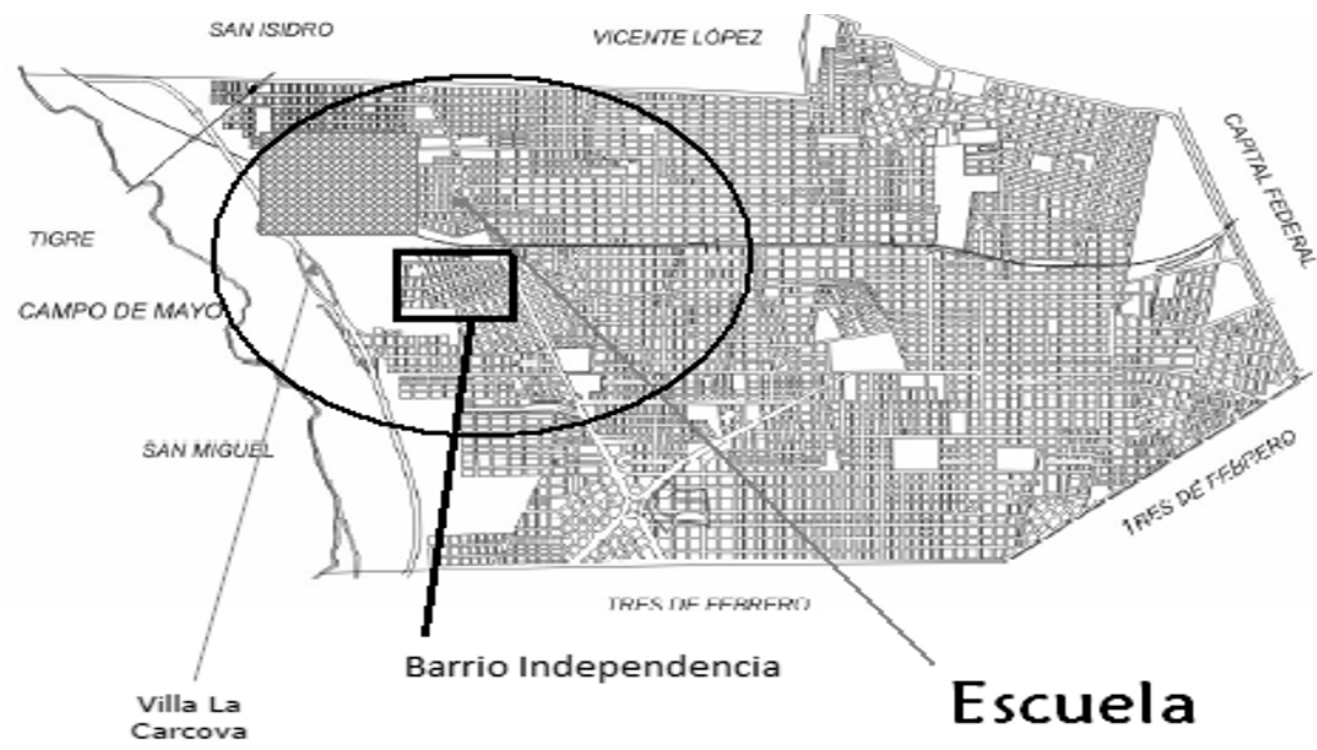

Fuente: Mapa realizado por estudiantes de sexto año de la escuela seleccionada en 2011. En el marco de un Taller Audiovisual. CEDESI-UNSAM ${ }^{4}$

En el diseño y desarrollo del trabajo de campo, la triangulación de los métodos de obtención y, por tanto, el análisis de datos, implicó trabajar con información de un rango de distintas unidades e informantes, tales como estudiantes de diferentes escuelas secundarias $y$, también, de distintos contextos. Es decir, escuelas secundarias con distintos IVSG 5 .

3. Las villas miseria en Argentina son los asentamientos informales ubicados en las periferias de las ciudades, caracterizados por una densa proliferación de viviendas precarias.

4. Centro de Estudios sobre Desigualdad, Sujetos e Instituciones (CEDESI) de la Universidad Nacional de San Martín.

5. Índice de vulnerabilidad socio geográfico creado por la Dirección de Planeamiento de la Provincia de Buenos Aires. Ver el Mapa Escolar (ABC). 
Esta utilización de una variedad de métodos se realiza para reducir el riesgo que las conclusiones reflejen solamente los sesgos sistemáticos o las limitaciones de un método específico (Maxwell, 1996). Así, la triangulación de la información se construyó sobre la base de encuestas semiestructuradas realizadas, observaciones y entrevistas individuales y grupales en profundidad, así como la información generada en los talleres de devolución y retroalimentación con supervisores, directores y docentes. Los métodos y técnicas de obtención de la información que guiaron este estudio, implicaron en un primer momento la aplicación de la encuesta a estudiantes en quince escuelas secundarias del partido de San Martín $y$, en un segundo momento, observaciones en profundidad de diferentes espacios escolares. Distintas materias y con distintos docentes, recreos, cantina, laboratorio, sala de informática, puerta de entrada y salida en una escuela secundaria de José León Suárez. Asimismo, para lograr variabilidad y contrastar la información obtenida con anterioridad, se realizaron entrevistas en profundidad y flash con estudiantes y docentes, así como entrevistas grupales con estudiantes para obtener una compleja trama de datos. Esas entrevistas sirvieron para referir y significar las prácticas de resistencias puestas en juego tanto dentro como fuera de las clases.

Es en este marco que se realizó la triangulación de las técnicas de obtención de información, porque se considera central para comprender los contextos de pobreza urbana en los que viven los estudiantes así como para conocer el ámbito institucional y a sus docentes para pensar, cómo los estudiantes producen sus discursos, dónde encuentran sus puntos de semejanza o ruptura, porque "las posiciones del sujeto se definen igualmente por la situación que a éste le es posible ocupar en cuanto a los diversos dominios o grupos de objetos" (Foucault, 2007, p. 72). A la vez, resultó relevante analizar las posiciones que los sujetos pueden ocupar, por ejemplo, en la red de las informaciones de una institución como la escuela. La caracterización de esa red de relaciones permitió dar cuenta de relaciones de saber, de poder y, por tanto, de resistencia en el ámbito escolar.

Por tanto, el análisis que se realizó implicó "colocarse a cierta distancia de este juego conceptual manifiesto" (Foucault, 2007, p. 81) e intentar determinar con qué esquemas podían estar ligados los enunciados unos con otros en un tipo de discurso, tratando de fijar "cómo pueden los elementos recurrentes de los enunciados reaparecer, disociarse, recomponerse, ganar en extensión o en determinación" (Foucault, 2007, p. 81). Se describieron las regularidades de las prácticas discursivas de los estudiantes a lo largo 
del trabajo de campo realizado, proponiendo redes conceptuales que se fueron construyendo en el proceso. Estas redes se fueron produciendo a partir de los enunciadores y sus relaciones de analogía, oposición o de complementariedad con otros enunciados como por ejemplo los de otros estudiantes, de sus docentes o de las autoridades escolares. Los discursos así concebidos constituyeron "un conjunto donde pueden determinarse la dispersión del sujeto y su discontinuidad consigo mismo" (Foucault, 2007, p. 75). Así, en el análisis tuvimos en cuenta siempre que los discursos que se caracterizan son prácticas (Goffman, 1995). Es decir, los sujetos producen sus discursos como todas sus prácticas en la urgencia de la situación inmediata, por tanto, esos discursos y/o prácticas siempre llevan las marcas de la situación en la que se han producido (Criado, 1998). Retomando a Scott (2000), el problema metodológico consiste en "¿cómo podemos evaluar el impacto de las relaciones de poder en los actos cuando el ejercicio de poder es casi constante?" (Scott, 2000, p. 50).

Entonces, las relaciones que el investigador fue estableciendo con los sujetos, fundamentalmente con los estudiantes pero, también, con docentes y autoridades escolares de la escuela secundaria donde se desarrolló el trabajo en profundidad, son centrales para pensar en las formas de producción científica en las ciencias sociales en la actualidad, en torno a estudios, no de las juventudes sino con los jóvenes. Una de las estrategias centrales de investigación de largo plazo en todo el proceso fue establecer confianza, conocimiento y compromiso con los otros. Se puede pensar que fue una estrategia que se volvió técnica de investigación indispensable.

Aquello que aquí se denomina la técnica del diálogo informal permite pensar desde lugares más cercanos a y con los sujetos -no objetos- de investigación. Conocer la vida y cultura cotidiana de los sujetos fue un proceso esencial; la relación que estableció el investigador con los distintos miembros de la investigación fue un proceso complejo y cambiante a lo largo de la investigación. Lograr empatía y confianza para caracterizar los procesos de resistencia y los sentidos que les otorgaban los sujetos en la cotidianeidad de la vida escolar, fue, justamente, un trabajo no sólo largo y profundo, sino de paciencia, minuciosidad, entendimiento, comprensión, etc.

Para construir relaciones de confianza y procurar comprender empáticamente a través de la solidaridad con las situaciones que vivían $\underline{y}$ aún viven ${ }^{6}$ estudiantes y docentes, se establecieron diálogos que 
fueron, en ocasiones, más importantes que los registros formales de obtención de información. Tal como dice Scott, "el efecto de la presencia de un maestro u maestra ante un grupo de estudiantes apenas se puede comenzar a medir cuando aquél o aquella sale del salón de clase o cuando los estudiantes salen al recreo" (Scott, 2000, p. 50). Los actos no dejan de ser opacos hasta que se pueda "hablar confidencialmente, tras bambalinas, con aquéllos cuyos motivos deseamos entender" Scott (2000, p. 50). Aquello fue lo central, la posibilidad de hablar y dialogar con los estudiantes y con los docentes. No entrevistar $y$, simplemente, obtener información.

Si bien los registros de entrevistas individuales, entrevistas flash y entrevistas grupales con los estudiantes se plantearon siempre como espacios de "libertad de expresión, lejos del alcance de los poderosos" (Scott, 2000, p. 50), buscar esos discursos ocultos que otorgan sentido a las prácticas de resistencia de estudiantes en contextos de pobreza urbana, implicó una técnica no formateada o guiada, tal como se estableció con las guías de entrevistas, por una comunicación que respete el lenguaje, los códigos, los saludos, las formas que los propios estudiantes tenían de ser y hacer y de los cuales el investigador tuvo que aprender?. Dialogar implicó conocer costumbres de los estudiantes como, por ejemplo aprender que, tal como dijo un estudiante, "dar la mano sin darla y luego golpear los puños", es signo de confianza, que dar un beso en el saludo es prácticamente ser un familiar, que las muestras de cariño y entendimiento no eran habituales, que no demuestran afecto si no se demuestra primero, etc. También, implicó que al ganar confianza, los estudiantes busquen y demanden al investigador por situaciones que se viven dentro de la escuela o fuera de la misma. Es decir, involucró profundizar las interacciones cotidianas con los estudiantes dentro y fuera de la escuela y con sus familias ${ }^{8}$, tal como expresa el siguiente registro de observación:

[En una salida a la universidad con estudiantes de quinto y sexto año de la escuela en la que iban a presentar cortos documentales en un festival de cine que allí se desarrollaba, se inició un diálogo con Ernesto. Era estudiante de sexto año. Comienza Ernesto preguntando por el auto que tenía y

contacto y relación con muchos de los estudiantes y docentes de aquel momento, aunque ya no como investigador.

7. Por ejemplo, se hace referencia a aprender los saludos que tienen los estudiantes en la escuela, o sus expresiones cotidianas.

8. Fundamentalmente, con sus madres. 
diciéndome que algunas veces me había visto en la puerta de la escuela subirme pero no podía identificar qué marca era].

Ernesto: ¿Fiat? ¿Renault? Ah, sí. Renault Focus.

Investigador: es Ford.

Ernesto: mi papá está por comprar su primer auto. Él está contento. Es un auto que hay que arreglar, cambiar algo del motor, arreglar las chapas. Es un Senda, un auto viejo.

Investigador: iqué bueno! ¿Estás contento?

Ernesto: sí, porque soy el menor y voy a poder manejar. Pero tengo que sacar el registro. Mi viejo también.

Entrevistador: ¿Cuántos años tiene tu papá?

Ernesto: 45. Capaz que algún día me lo presta para venir a la universidad. ¿Cómo fue su primer día de clases en la universidad?"'. [Me hace esa pregunta porque él en otra ocasión, en una hora de clase ya había preguntado si yo había estudiado, qué, dónde y qué significaba].

Ernesto: ¿Fue como el primer día en la secundaria? ¿Algo nuevo o desconocido?

(...)

Ernesto: no me imagino estudiando, pero sé que está cerca. Me pone un poco nervioso todo eso. Toda esa novedad y todo lo nuevo.

Entrevistador: ¿Qué tal te viene venir desde tu casa a esta universidad?

Ernesto: bien, el tren me deja a algunas cuadras. Son cuadras medio peligrosas, pero todo bien. Es rápido. Quizás, me presta el auto mi viejo y voy a invitar chicas que se suban.

[Luego de este comentario empezamos a bromear un poco al respecto de las novias que puede tener él con un auto].

Ernesto: ¿Usted estuvo con chicas en la universidad mientras estudiaba?

(...).

Ernesto: yo también quiero estar con chicas.

(Registro de observación, diálogo informal con estudiante de sexto año, 26 de septiembre de 2012).

Si bien el investigador ya tenía confianza con el estudiante, este diálogo permitió conocer algo de su historia familiar, algo de sus ideas del futuro,

9. Al ser un diálogo, por supuesto, que no está mediado por ningún artefacto de grabación y de tan pendiente por recordar aquello que el estudiante decía, contaba y preguntaba la memoria jugó una mala pasada y no se pudo reconstruir ni inmediatamente después ni nunca más, algunas de las respuestas que fui haciendo a Ernesto. Por tanto, se presenta un registro fragmentado, priorizando las respuestas de Ernesto. 
sus miedos, sus intereses. Significó un espacio informal para hablar, no para preguntar y responder. No había entrevistado ni entrevistador sino dos personas hablando. El estudiante de hecho era el que más preguntaba acerca de la vida y a través de la respuesta, él se expresaba acerca de la suya. El diálogo continuó así, como también, la relación de confianza con el estudiante hasta la actualidad, porque de hecho el estudiante al ser egresado de la secundaria e ingresar a estudiar a la universidad, busca como referente para hablar y como pedido de ayuda, entre otros, al investigador que ya no cumple más ese papel con esta persona y excede todos los límites que tuvo la investigación. Conocer algunos aspectos de las historias y las formas de vida de los estudiantes presupone una relación de confianza entre entrevistador y entrevistado y es por ello que la investigación es una búsqueda típicamente abierta que "induce al analista a practicar esa virtud de la humildad" (Ferrartotti, 2007, p. 27) esencial para cualquier científico. Es necesario agregar que esa humildad implica, necesariamente, entender con exactitud que en esos momentos, se deja de ser entrevistador y el otro entrevistado, investigador e investigado. Hablar con este estudiante de esta forma permitió caracterizar y analizar sus prácticas en el aula de otra manera. Entender por qué hacía lo que hacía en el aula y cuáles eran sus motivos más profundos. Por qué con un docente se comportaba de una forma y con otro de otra forma. Saber que quería estudiar en la universidad e ir con el posible auto que su papá le podía llegar a prestar, si es que llegaba a comprarlo y a arreglarlo, permitió entender que este estudiante, si se comportaba de una manera "no adecuada" con algún docente no era porque no tenía ganas de estudiar y aprender, tal como muchas veces se dice y circula, sino que esa práctica de resistencia adquiría otro sentido y otra característica en este joven.

La construcción de confianza, y los diálogos informales, no se realizó solamente con estudiantes sino, también, con docentes. En muchos momentos de las observaciones realizadas, el investigador se ponía en el lugar de escucha, como descarga ante clases en las que los estudiantes les respondían, reaccionaban o no hacían la tarea que ellos les pedían, tal como se expresa en muchos registros a lo largo de la investigación doctoral (Langer, 2014). Así, retomar diálogos privados de docentes con el investigador luego de esas clases tensas que habían tenido con sus estudiantes, permitió significar el otro lado de las prácticas de resistencia. Es decir, permitió describir las regulaciones o el intento de regular las conductas por parte de los docentes hacia los estudiantes, tal como se retoma a continuación algunos ejemplos: 
Es un atrevido, un mocoso, no maneja la ira. Es vago. Puede dar mucho más. Se piensa que es el profesor. Me quedo cargada cuando pasan estas cosas. Si fuera buen compañero.... Tiene capacidad pero tiene otras falencias. Te dan ganas de reventarlo. Yo sé que tiene un padre muy agresivo entonces no puedo hablar con él porque quizás lo agarra, entonces no sirve.

(Registro de diálogo con docente post-clase, Sexto año, 15 de septiembre de 2011).

Estoy cargado, como sin palabras, no sé qué decir, no sé qué hacer. No me puedo enfrentar a todo el curso, entonces lo que intento hacer es hablar uno por uno. Por ejemplo, Leonel es un líder negativo, quien sutilmente es el que revoluciona todo el curso. No como los otros chicos que hablan y aunque me dicen de todo se puede trabajar. ¿Qué se puede esperar de esos chicos que no tienen límites en sus propias casas? Hay un grupo de chicos que yo ya no espero nada de ellos. Desde el primer día que no espero nada. Les puse la nota más baja y no un aplazo para darles una oportunidad pero ellos no reaccionan, hacen la suya. No hacen nada. ¿Qué puedo esperar de ellos? Seguro te vas con una muy mala impresión de la clase de hoy... [EI profesor dice todo esto y está nervioso, le tiemblan un poco las manos].

(Registro de diálogo con docente post-clase, tercer año, 7 de Julio de 2011).

Lejos de querer significar aquellos discursos docentes desde las clasificaciones y/o estigmatización, se hizo desde los atributos y las formas que tenían para poner en palabras y expresarse sobre las reacciones que los estudiantes tenían en una clase. Se abrió un espacio para que puedan enunciar algo que, en general, los docentes no pueden hacer, porque no tienen espacios para hacerlo. Los dos registros presentados más arriba, tienen la semejanza de docentes que dicen estar y quedarse cargados ante incidentes en sus clases. El investigador se constituye, también, en una persona con quien pueden descargar, pensar sus cotidianos, sus actividades, sus quehaceres buenos y malos, las tensiones y contradicciones en las que viven sus estudiantes y ellos todos los días, ante sinsentidos que se suceden entre las normativas o regulaciones escolares y lo diario. Eran docentes que se quedaban sin herramientas para trabajar con jóvenes que viven realidades muy crudas. Ello expresa las contradicciones entre el sistema educativo que deja sin recursos a las escuelas y a los docentes para 
regular y sancionar las conductas de sus estudiantes y las perspectivas que los jóvenes muestran para educarse en un contexto en el que saben que "da lo mismo estudiar" porque igual no van a encontrar trabajo.

En este sentido, el diálogo implicó construir confianza con los interlocutores a partir de la escucha y de erigir una relación significativa, "una auténtica interacción, que, en tanto involucre de manera natural a las personas sobre las que se conduce la investigación, reclame al investigador permanecer en la causa y derribar el muro defensivo tradicionalmente colocado" (Ferrarotti, 2007, p. 17). Construir relaciones de confianza implicó una perspectiva política porque involucró una producción y una conducta metodológica rigurosa del investigador con los otros sujetos. Comienza con un acto del investigador hacia el sujeto, aún más cuando son jóvenes, y luego se convierte en un ida y vuelta entre ambos. Tal como dice Cornu (1999), la confianza en la relación pedagógica "es una renuncia liberadora a un poder absoluto" (1999, p. 23) a la vez que abre un campo de acción a aquel que es menos fuerte.

Generar confianza con estudiantes y docentes, también, implicó en los primeros momentos compartir los registros, asegurarles que ninguno de sus nombres figuraba en ellos, que eran confidenciales y anónimos. Por ejemplo, cuando se observaron las clases de primer año, al principio hubieron interpelaciones de los estudiantes, por aquello que el investigador escribía, indagando si ellos estaban dentro de esa escritura o no, si se escribía o no sobre las cosas feas que ellos estaban diciendo en clase. A pesar de las explicaciones, siempre escasas para ellos, los estudiantes querían preguntar más acerca de la finalidad de la investigación, siempre. Una estudiante de primer año en una de las primeras observaciones de clase, preguntó: "¿es para cerrar la escuela?" (Registro de diálogo informal con estudiante, 2011). Pregunta significativa en un contexto en donde todo se cierra, lo que falta son los lugares, nada perdura y en el que los estudiantes ven la condena social que hacia ellos se les impugna. También, esto se manifestó en otro estudiante ante una situación en la que lo sacan del aula por portarse mal y que luego al preguntarle qué fue lo que paso contesta:

Cuando no viene usted la profesora dice que tiene vergüenza de cómo nos portamos. Los profesores tienen vergüenza, los profesores tienen vergüenza 
En cambio, los estudiantes de sexto año interpelaban de otras formas, haciendo al investigador partícipe de las dinámicas de la clase tanto para ejercicios o actividades que tenían que hacer así como de las dinámicas que se armaban a partir de las confusiones que se generaban en la clase. Por ejemplo ante la confusión que había en una clase algunas estudiantes interpelaban preguntando:

¿Ya se volvió loco?, ¿No se la quiere llevar [a la profesora]?, ¿Por qué no fue al geriátrico ${ }^{10}$ ?

(Registro de observación de clase, Sexto Año, 13 de octubre de 2011).

Es en función de estos diferentes modos de acercarse y percibir la presencia del investigador en el aula, que surge la pregunta de cómo esa presencia podría variar o modificar la conducta o no de los estudiantes. Interrogante central en cualquier investigación en ciencias sociales que quiera describir determinadas prácticas tal como suceden en la realidad. Así, a través del siguiente registro de observación de clase se procura discutir, justamente, sobre las posibilidades y límites de la técnica de observación de los estudiantes en el aula porque, de hecho, producto del siguiente registro de observación se decidió aplicar, también, otras técnicas de obtención de información:

[Karina e Inés le dicen a la profesora que me digan a mí lo que ella dijo de la actividad de redacción que hicieron con algunas palabras que les dio para hacer]. Karina: dígale lo que usted dijo de mi voz. [La profesora sigue copiando las preguntas en el pizarrón de una nueva actividad].

Inés: sí dígale de las patadas en las pelotas. Me dijo que soy una patada en las pelotas.

Alicia [parada sobre un escritorio pintando un grafiti que dice "egresa2"]: Profe, no se ilusione con el pizarrón. [La profesora termina de escribir].

Karina: lea lo que escribimos de cuando está él observando. Léalo profe, no tenga vergüenza.

(Registro de observación de clase, Sexto año, 6 de octubre de 2011).

Son las estudiantes que interpelan a la profesora de una clase que se observó sistemáticamente durante todo 2011 para que exponga aquello

10. Se referían irónicamente a una actividad que hicieron los estudiantes de sexto año para estudiar a una organización fuera de la escuela. 
que escribieron sobre las observaciones que se realizaron. En este marco de interpelación de los estudiantes, dimensión que se constituye en unos de los rasgos que adquieren las prácticas de resistencia de estudiantes, es que la profesora cuenta la actividad que les hizo hacer a los estudiantes en torno a la presencia del investigador en el aula porque ella sentía que cuando estaba se modificaba el clima de la clase, que los comportamientos eran diferentes. Aunque aquí sea objeto de reflexión, la preocupación de la docente era saber qué les pasaba a los estudiantes con la visita del investigador. Así la docente, comparte esa actividad mostrando una hoja que dice "Observador - escuela - móvil - palabra - silencio" que fueron las palabras con las que les propuso pensar a los estudiantes. Al respecto sus respuestas escritas fueron las siguientes:

-Psicólogo- sociólogo - acosador - expresan los silencios.

- Muchas cosas, no incomoda pero sí a algunos incomoda porque escribe TODO lo que hacemos en el aula.

- Los jueves estamos un poquito alterados, cuando no está el observador con la profe estamos más tranquilos y más en silencio.

- Proyecto para la universidad, se sienta en frente en silencio para observar el comportamiento de cada uno de nosotros.

- Nunca habla, no me molesta pero anota cada cosa que decimos, ¿para qué sirve anotar todo lo que decimos y hacemos?

- Con la visita de Eduardo nos ponemos más locos, observa toda la clase por completo y anota todo lo que los alumnos realizamos con normalidad.

- Mira, analiza, piensa resolver una situación.

- Concurrimos con normalidad.

- El silencio nos da paz a veces pero a veces es inquietante. Eduardo está siempre en silencio observando lo que pasa en cada clase.

- Observar el comportamiento de los alumnos en la clase, escribe muchas palabras.

- Al principio, los primeros días molestaba un poco su presencia pero ya nos acostumbramos y nos da lo mismo si está o no está. Como siempre está en silencio, es como si no estuviera, nos comportamos como si estuviéramos solos con la profesora.

- Observa a determinadas personas. También puede observar una sola en distintas ocasiones. Es un hombre, observa la vida de los adolescentes en el aula. Cuando viene cambia un poco el clima. 
-Ahora es como un alumno más, pero a veces se pone la situación incómoda cuando se arman líos.

-Es una buena persona dentro de todo. Eduardo observa también otras aulas. Atento. Simpático. Inteligente. Audaz.

-Su silencio nos intriga, saber qué anota, si nos paramos a veces lo escribe, si gritamos lo escribe y si hablamos entre nosotros también. Para mi describe la situación de los chicos hoy, los adolescentes en el colegio. Si hablan con lenguaje adecuado, etc.

-A mí el silencio me incomoda a veces pero a veces es bueno porque te hace reflexionar sobre uno mismo y el entorno.

(Documento de la escuela, Actividad de Sexto año, 6 de octubre de 2011).

La variabilidad de aquello que dicen los estudiantes de este curso con respecto a las observaciones va desde:

"Nos ponemos más locos" o "estamos un poquito alterados" a "nos da lo mismo si está o no está", "es como si no estuviera" o "ahora es como un alumno más"

(Documento de la escuela, Actividad de Sexto año, 6 de octubre de 2011).

Estos registros sobre aquello que pasa en el aula con los estudiantes cuando está el investigador, es un proceso de reflexión que en ningún momento ponen en cuestión esa situación. Quizás, lo más cercano a ello es la pregunta que allí aparece:

¿Para qué sirve anotar todo lo que decimos y hacemos?

(Documento de la escuela, Actividad de Sexto año, 6 de octubre de 2011).

Aunque es una pregunta que tampoco pone en cuestión la presencia del investigador, sino aquello que hace, incluso ese proceso de reflexión permite describir en alguna medida cómo los estudiantes se ven a sí mismos o qué sienten en esos momentos y, por tanto, fue un registro que permitió por un lado validar la información de las observaciones realizadas en ese curso, a la vez que tener ciertos recaudos metodológicos a la hora de analizar la información obtenida. Por ejemplo, algunos estudiantes de sexto año decían que varía su comportamiento o el clima con la presencia del investigador $y$ otros que se fueron acostumbrando. 
Lo que no hizo el investigador, fue ignorar que estas apreciaciones sobre su presencia en el campo se construían e incluso influían en la propia construcción del objeto de estudio porque "nuestros interlocutores nos confieren un lugar en su mundo; y es a partir de este posicionamiento impuesto que nosotros construimos nuestra perspectiva" (Althabe y Hernández, 2005, p. 82). Como investigadores no se puede ignorar esos posicionamientos, identificaciones y roles que los propios informantes asignan. Es por ello que en algunas entrevistas individuales a estudiantes, el investigador fue preguntando qué pensaban sobre la observación y si los modificaba o no esa presencia, para poder tener control sobre la información a analizar. Con respecto a la modificación de los comportamientos o no de los estudiantes, nuevamente dimensión central en este estudio, los estudiantes respondieron:

Ernesto: "no sé, mirando cómo se portan los pibes. Yo qué sé. Antes nos retaba de nada así. Así no nos gritaba tanto".

Investigador: "o sea ¿pensás que el hecho de que yo esté ahí observando cambia algo o no en la profesora?".

Ernesto: "sí. Si después nos decía que nos teníamos que portar bien antes que venga. Que no la podemos hacer pasar vergüenza, nada de eso".

Investigador: "¿y tuyo cambió algo o no cada vez que yo venía?".

Ernesto: "no, yo seguía siendo igual. Lo mismo".

Investigador: "¿vos seguías siendo igual?".

Ernesto: "si".

(Entrevista a estudiante, varón, 14 años, 27 de octubre de 2011).

Investigador: "¿te gustó o no que venga?".

María: "sí. Me dio igual".

Investigador: "¿y vos te portabas igual o te portabas distinta cuando yo venía?".

María: "no, casi igual. A veces distinto".

Investigador: "¿por qué a veces distinto?".

María: "no me portaba tan mal, igual. Me daba igual".

(Entrevista a estudiante, mujer, 13 años, 27 de octubre de 2011).

Investigador: "¿qué pensás que estuve haciendo todo este año en el aula?". 
Mauricio: "cómo se portan cada uno y eso".

Investigador: "¿a vos te modificaba en algo o no que yo venga?".

Mauricio: "no".

Investigador: "¿te daba igual?"

Mauricio: "si".

También, desde estos registros de entrevistas, se consideraron las modificaciones en las formas que los docentes, muchas veces, encontraban para sancionar y controlar las conductas de diferentes formas cuando el investigador estaba o no presente. De esta forma, fue interesante indagar con los docentes cómo esos cambios de conducta para sancionar o controlar suponían o no ciertas imágenes de un deber ser de la conducta. A la vez, se introdujo un signo de interrogante -en términos de un recaudo metodológico y de mecanismo de control con la información obtenida- al respecto de cómo se presentaban las formas de sancionar las conductas en las clases y de cómo variaban o no esas formas según los días de observación y según el momento del año en el que se observaba.

Por tanto, otra dimensión central para los diálogos con estudiantes y docentes en las escuelas fue el tiempo. Haber comenzado en 2009 y continuar hasta el momento que se está escribiendo este escrito, el trabajo de campo, investigación y transferencia con la escuela, permitió establecer cada vez mayor caudal de conocimiento -no ya informaciónrespecto de la realidad social de la escuela y de los sujetos que en ella participan, y en tanto más conocimiento se genera, el investigador se sensibiliza en mayor medida para registrar acontecimientos que de otro modo no podría. También, esta mayor sensibilidad por la institución, por los docentes y por los estudiantes puede establecer límites fuertes al momento de analizar y que fueron tenidos muy en cuenta como una estrategia de control al momento de la escritura y de la delimitación del objeto de investigación. En este sentido, las investigaciones como la que aquí se presenta, "tienden a hacer emerger, desde abajo en contacto directo con el objeto de investigación (observaciones participantes, investigación de fondo), las áreas problemáticas y los eventuales conceptos operativos" (Ferrarotti, 2007, p. 25).

Se desarrolló un trabajo de investigación a la vez que de transferencia porque desde el 2009 se implementa un taller audiovisual en la misma 
escuela. Desde sus inicios, el taller se planteó como una actividad colectiva y en conjunto entre un grupo de docentes e investigadores de la universidad y docentes de la escuela secundaria, como un espacio de extensión y para generar instancias de reflexión y debate en torno a las experiencias de escolarización en contextos de pobreza urbana. En este espacio, se propone como uno de los objetivos la producción de audiovisuales por parte de los estudiantes, con el fin de propiciar espacios de pensamiento y problematización de la realidad a través de la realización documental, a la vez que generar debates e intercambios de las problemáticas barriales en el espacio escolar (Carpentieri, Dafunchio, Langer y Machado, 2015). Esto es, que la escuela devenga no sólo un lugar donde estar, sino un lugar donde pensar y problematizar la vida social e individual. Se procura propiciar espacios de reflexión en la lógica escolar a través de los cuales los adolescentes y jóvenes puedan desde su mirada caracterizar su cotidianeidad escolar y barrial que suele ser silenciada y ocultada.

El taller como una propuesta pedagógica dentro de la escuela implica no sólo la generación de saberes en los estudiantes sino, también, la posibilidad de pensar de otro modo las relaciones entre profesores y estudiantes. Hablar, decir, contar y expresar se vuelven claves centrales en la realización documental que, por otra parte, permite ubicar en la historia las microhistorias que suelen atravesar los sujetos que viven en las villas del conurbano bonaerense. Se genera un espacio en que palabra y acción, siguiendo a Arendt (1993), se vuelvan moneda corriente, en el que los estudiantes aparecen como sujetos sociales, relatando su historia y pensándose en ella. Por ello, el eje del taller está centrado en la vida cotidiana de los estudiantes en tanto productores de relatos para ser proyectados.

De forma que la implicación y la confianza en todo este proceso y con todas las técnicas puestas en juego -sea entrevistas formales, entrevistas flash, diálogos, observaciones participantes o el taller audiovisual mismo- fue fundamental para lograr empatía, familiaridad e intimidad con los sujetos, para poder comprender sus acciones a partir de aquello que piensan y por aquello que están viviendo. El interés por aquello que mostraban, demostraban, decían o hacían tanto docentes como estudiantes, fue una estrategia central puesta en juego siempre en todos los registros. Pero esto no fue actuar (Bourdieu, 1999) sino que fue parte del ser en la investigación y en la vida, fue y es una creencia porque "hay una inversión emocional de los sujetos en el juego de la 
interacción: porque lo que está en juego no es solamente el crédito social que podemos obtener, sino toda nuestra identidad" (Criado, 1998, p. 61).

\section{A modo de cierre: investigaciones de largo plazo y comprometidas con las vidas de los jovenes}

El diseño de la investigación doctoral realizada fue un proceso social (Jelin, Llovet y Ramos, 1996) en el que la problemática, en este caso las prácticas de resistencia de estudiantes en contextos de pobreza urbana, se fue elaborando y transformando a lo largo de la práctica misma de la investigación. Los resultados no fueron más que un momento del proceso continuo en el cual se van redefiniendo las categorías o replanteando las preguntas. En tanto la realidad social está, también, en proceso de continuo cambio se comprende la problemática en estudio en función de procesos sociales que se desarrollan en el tiempo, en el espacio y en la historia. Si la realidad va mutando a medida que cambian las condiciones sociales, materiales y/o culturales de existencia, el desafío, entonces, pasa por poner en juego una propuesta metodológica innovadora, comprometida, compleja, científica, política y posible de largo plazo.

La caracterización de las prácticas de resistencia de estudiantes en contextos de pobreza urbana y la regulación de las conductas tuvo la complejidad de tener que meterse en la caja negra (Karabel y Halsey, 1976) de la escuela, en las clases y tratando de construir confianza con los estudiantes, los docentes, las autoridades escolares, ser parte de la institución para que la información obtenida sea significativa, válida y tenga confiabilidad. Supuso conocer la vida de la institución y las formas de ser de los sujetos. A la vez, supuso conocer la vida de los sujetos fuera de las instituciones.

Caracterizar las prácticas de resistencia de estudiantes en contextos de pobrezaurbana demandó adoptar diferentes metodologías y dimensiones de investigación apostando a nuevas formas de acercamiento a lo social, en contra de las ideas de neutralidad social y/o valorativa en la investigación, que permitan construir una mirada socioeducativa creativa de las escuelas en general y de los sujetos, los jóvenes en su relación con los adultos, en particular. 
De esta forma, las estrategias que se propusieron permiten comprender la formalidad de las prácticas, a través de los comportamientos, los intereses y los deseos de los estudiantes que, como dicen Deleuze y Guattari (1988), son la base de toda sociedad que se crean, se agotan o mutan y que se suman, se substraen o se combinan en una investigación de largo plazo y comprometida en torno a los sujetos y a los lugares (instituciones) con los cuáles se trabaja, no sólo en un proceso de obtención de información sino, también, realizando transferencia, apoyando a las instituciones y a los sujetos como parte esencial de la metodología de investigación utilizada. En este sentido, fueron centrales las "retroalimentaciones" (Sirvent, 1999, p. 61) de la información que se fue obteniendo a lo largo de los años, a la vez que trabajar junto con las instituciones. Estos son espacios desde los cuales pensar e intervenir en torno a la desigualdad educativa y social de una forma general y, también, en torno a las particularidades y obstáculos institucionales con los que se enfrentan cotidianamente en la escuela.

Esta estrategia metodológica comprometida, compleja y de largo plazo se construyó a partir de las siguientes acciones en profundidad tales como: participar de la vida diaria de la comunidad sea escolar u otra; un trabajo de campo profundo de largo plazo y que duró la cantidad de años suficientes para conocer a los sujetos y para hacerse conocer; compromiso con aquello que los propios actores de la escuela proponían con la inserción de la investigación; que las reflexiones de investigación constituyeran aportes para producir mejoras en la dinámica escolar; acciones de transferencia con la institución escolar; estar siempre del lado de investigador comprometido y participante tratando, a la vez, de lograr una separación con el objeto de estudio.

Para abordar de una forma compleja y comprometida el objeto de estudio, dar sentido o interpretar los fenómenos en los términos del significado que las personas les otorgaban, la investigación abarcó métodos, estrategias y técnicas de "una variedad de materiales empíricos - estudio de caso, experiencia personal, introspectiva, historia de vida, entrevistas, textos observacionales, históricos, interaccionales y visuales- que describen los momentos habituales y problemáticos y los significados en la vida de los individuos" (Vasilachis de Gialdino, 2007, p. 24). Abordar las prácticas de resistencia de estudiantes implicó interesarse y comprometerse por las formas en las que el mundo social era interpretado, comprendido, experimentado y producido tanto por estudiantes como por docentes en las escuelas, sensibilizarse por el contexto social en el que se producen, 
tratar de conocer las dinámicas de esos procesos sociales (Vasilachis de Gialdino, 2007). Para ello, fue imprescindible la inmersión en la vida cotidiana de la situación seleccionada para el estudio, la valoración y el intento por descubrir la perspectiva de los actores sobre sus propios mundos y la consideración de la investigación como un proceso interactivo entre el investigador y esos participantes como descriptiva y analítica, que privilegia las palabras de las personas y su comportamiento observable como datos primarios. Fue, principalmente, esforzarse para "hacer hablar a los otros" (Iniesta y Feixa, 2006, p. 3) intentando establecer con ellos una relación de confianza, es decir "aceptar implicarse, estudiarlo a través de una empatía que sólo heurísticamente admite una distancia entre investigador e investigado" (Iniesta y Feixa, 2006, p. 7), ello desde una actitud y un compromiso político del investigador con los sujetos y los contextos en lo que se enmarca la investigación.

\section{Referencias bibliográficas}

Althabe, G. y Hernández, V. (2005). Implicación y reflexividad en antropología. En Hernández, V., Hidalgo, C. y Stagnaro, A. (comps.). Etnografías globalizadas (pp. 71-90). Buenos Aires: Sociedad Argentina de Antropología.

Arendt, H. (1993). Entre el pasado y el futuro. Ocho ejercicios sobre la reflexión política. Barcelona: Ediciones Península.

Arroyo, M. (2001). Educación en tiempos de exclusión. En Gentili, P. y Frigotto, G. (comp.), La ciudadanía negada. Políticas de exclusión en la educación y el trabajo (pp. 266-275). Buenos Aires: CLACSO.

Bonal, X. (1998). Sociología de la educación. Una aproximación crítica a las corrientes contemporáneas. Barcelona: Paidós.

Bourdieu, P. (1999). La miseria del mundo. Buenos Aires: Fondo de Cultura Económica.

Carpentieri Y., Dafunchio S., Langer E. y Machado M. (2015). Producir saberes desde la experiencia de un taller audiovisual en una escuela secundaria en contextos de pobreza urbana. Novedades Educativas, 294, 27-32.

Cornu, L. (1999). La confianza en las relaciones pedagógicas. En Frigerio, G. (ed), Construyendo un saber sobre el interior de la escuela (pp. 19-26). Buenos Aires: Novedades Educativas.

Criado, M. E. (1998). Los decires y los haceres. Papers, 56, 57-71. 
Cruikshank, B. (1996). Revolutions within: self-government and self-esteem. En Barry, A., Osborne, T., Rose, N. (eds.), Foucault and political reason. Liberalism, neo-liberalism and rationalities of government (pp. 232-248). London: UCL Press.

Deleuze, G. y Guattari, F. (1988). Mil Mesetas. Capitalismo y esquizofrenia. Valencia: Pre - Textos.

Ferrarotti, F. (2007). Las historias de vida como método. Convergencia, 14(44), 15-40.

Foucault, M. (2007a). La arqueología del Saber. Buenos Aires: Siglo Veintiuno editores.

Foucault, M. (2007). Nacimiento de la biopolítica. Buenos Aires: Fondo de Cultura Económica.

Gatti, G. (2004). La teoría sociológica visita el vacío social. Madrid: Centro de Estudios sobre la Identidad Colectiva/ Universidad del País Vasco.

Goffman, E. (1995). Estigma. La identidad deteriorada. Buenos Aires: Amorrortu Editores.

Grigñon, C. y Passeron, J. C. (1991). Lo culto y lo popular. Miserabilismo y populismo en sociología y en literatura. Buenos Aires: Nueva visión.

Grinberg, S. (2008). Educación y poder en el siglo XXI. Gubernamentalidad y pedagogía en las sociedades de gerenciamiento. Buenos Aires: Ed. Miño y Dávila.

Iniesta, M. y Feixa, C. (2006). Historias de vida y ciencias sociales. Entrevista a Franco Ferrarotti. Perifèria. Revista de Recerca i Formación en Antropologia, 5, 1-14.

Jelin, E., Llovet, J. y Ramos, S. (1996). Un estilo de trabajo: la investigación microsocial. En Corona, R. (ed), Problemas metodológicos en la investigación sociodemográfica ( $p p$. 1-17). México: PIAPAL / El Colegio de México. Disponible en: http://www.sitiosur.cl/r. php?id=271.

Karabel J. y Halsey A. H. (1976). La investigación educativa: una revisión e interpretación. Poder e ideología en educación, pp. 1-33. Nueva York: Oxford University Press.

Kuasñosky, S. y Szulik, D. (1995). La barra de la esquina: violencia y exclusión social. Delito y Sociedad. Revista de Ciencias Sociales, 4(6/7), 165-170.

Langer, E. (2014). Dispositivos pedagógicos en las sociedades de control: prácticas de resistencia de estudiantes y regulación de las conductas en escuelas secundarias en contextos de pobreza urbana del Partido de San Martín. (Tesis inédita de doctorado). Buenos Aires: Universidad de Buenos Aires/ Facultad de Filosofía y Letras.

Mallimaci, F. y Salvia A. (coord.) (2005). Los nuevos rostros de la marginalidad. La supervivencia de los desplazados. Buenos Aires: Editorial Biblos.

Maxwell, J. A. (1996). Qualitative research design. An Interactive Approach. London: Sage Publicatios. 
Miller, P. y Rose, N. (1990). Governing Economic Life. Economy and Society, 19(1), 1-31.

Rose, N. y Miller P. (1992). Political power beyond the State: problematics of government. The British Journal of Sociology, 43(2), 173-205.

Scott, J. (2000). Los dominados y el arte de la resistencia. Discursos ocultos. México: Ediciones Era.

Sirvent, M. T. (1999). Cultura popular y participación social. Una investigación en el Barrio de Mataderos (Buenos Aires). Buenos Aires: Miño y Dávila Editores.

Torres C. A. (2008). Teoría crítica y sociología política de la educación: argumentos. En Mora-Ninci, C. y Ruíz, G. (comps), Sociología política de la educación en perspectiva internacional y comparada. Las contribuciones de Carlos Alberto Torres (pp. 133-180). Buenos Aires: Miño y Dávila Ediciones.

Vasilachis de Gialdino, I. (2007). Estrategias cualitativas de investigación. Buenos Aires: Gedisa. 